\title{
Preparation of enantiomerically pure anti-1,3-diols by sequential ruthenium-mediated asymmetric hydrogenation reactions
}

\author{
Olivier Labeeuw, Jean-Baptiste Bourg, Phannarath Phansavath, and Jean-Pierre Genêt* \\ Laboratoire de Synthèse Sélective Organique et Produits Naturels, UMR CNRS 7573, ENSCP, \\ 11 rue Pierre et Marie Curie, 75231 Paris Cedex 05, France \\ E-mail: jean-pierre-genet@enscp.fr
}

\section{Dedicated to Professor Alain Krief on the occasion of his $65^{\text {th }}$ birthday}

\begin{abstract}
A ruthenium-mediated sequential approach to anti-1,3-diols is described. A series of enantiomerically enriched 1,3-diols has been synthesized from $\beta$-keto esters using rutheniummediated asymmetric hydrogenation followed by diastereoselective hydrogenation of the resulting $\beta$-hydroxy ketones, obtained via the corresponding Weinreb amides. Using this sequence, diversely substituted anti-1,3-diols were obtained in good yields with a very high level of enantio- and diastereoselectivity (ee and de up to $99 \%$ ).
\end{abstract}

Keywords: 1,3-Diols, asymmetric hydrogenation, ruthenium catalysts, atropisomeric ligands

\section{Introduction}

Because of the prevalence of 1,3-dioxygenated substructures in biologically active natural products such as polyether and polyene macrolide antibiotics, ${ }^{1}$ the stereoselective synthesis ${ }^{2}$ of these moieties is of particular interest. Besides enzymatic routes ${ }^{3}$ for stereoselective preparation of the 1,3-diol motif, metal hydride reduction of 1,3-diones ${ }^{4}$ and transition metal-catalyzed hydrogenation reactions of 1,3-diketones ${ }^{5}$ and 3,5-dioxoesters ${ }^{6}$ have been reported. Recently, a general approach to syn and anti-1,3-diols using Jacobsen's hydrolytic kinetic resolution method

has been described. ${ }^{7}$ Apart from these methods, the stereoselective reduction of $\beta$-hydroxy ketones has been extensively studied, affording either $s y n$-1,3-diols by using $\mathrm{Et}_{3} \mathrm{~B} / \mathrm{NaBH}_{4}{ }^{8}$ and $\mathrm{Et}_{2} \mathrm{BOMe} / \mathrm{NaBH}_{4}{ }^{9}$ combinations, or anti-1,3-diols by using $\mathrm{Me}_{4} \mathrm{NBH}(\mathrm{OAc})_{3} .{ }^{10}$ However, these boron reagents are usually employed in stoichiometric or excess quantities, generating considerable amounts of waste. Therefore, a catalytic sequential route to syn or anti-1,3-diols through diastereoselective hydrogenation of $\beta$-hydroxy ketones using ruthenium complexes would be of synthetic utility. As part of our work towards the total synthesis of biologically 
relevant natural products using ruthenium-mediated asymmetric hydrogenation as a key step, ${ }^{11}$ we were interested in developing an efficient and general catalytic route to a variety of functionalized enantiomerically enriched anti-1,3-diols 4 by using sequential hydrogenation reactions $^{12}$ of $\beta$-keto esters $\mathbf{1}$ and of the resulting $\beta$-hydroxy ketones $\mathbf{3}$ as depicted in Scheme 1.<smiles>[R]C(=O)CC(=O)[O-]</smiles>

1

$\mathrm{H}_{2} \downarrow\left[\mathrm{RuL}_{n}^{*}\right]$<smiles>[R]C(O)CC(=O)OCC</smiles>

2

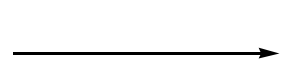<smiles>[R]C(O)C([R])C([R])O</smiles>

$\mathrm{H}_{2} \uparrow\left[\mathrm{RuL}_{n}{ }^{*}\right] \quad \mathrm{L}^{*}=(R)-\mathrm{MeO}-$ BIPHEP
or $(R)-$ SYNPHOS

1. $\mathrm{MeO}(\mathrm{Me}) \mathrm{NLi}$

2. $\mathrm{R}^{2} \mathrm{Li}$<smiles>[R]C(=O)CC([R])O</smiles>

3

\section{Scheme 1}

Hence, depending on the configuration of the chiral ligand, asymmetric hydrogenation of $\beta$ keto esters 1 would easily deliver both enantiomers of 2, while a variety of enantiomerically enriched $\beta$-hydroxy ketones $\mathbf{3}$ would be prepared from $\mathbf{2}$ by addition of alkyl or aryl lithium reagents onto the corresponding Weinreb amides. Ruthenium-catalyzed diastereoselective hydrogenation of compounds 3 would then afford enantiomerically enriched syn or anti-1,3diols.

\section{Results and Discussion}

Thus, a variety of enantiomerically pure $\beta$-hydroxy ketones $\mathbf{3}$ were prepared from $\beta$-keto esters $\mathbf{1}$ (Scheme 2, Table 1). A series of enantiomerically enriched $\beta$-hydroxyesters 2 was first synthesized via asymmetric hydrogenation of $\beta$-keto esters 1. Hydrogenation reactions of compounds 1a-1d were performed under optimized conditions using either the $\mathrm{RuCl}_{3} /(R)-\mathrm{MeO}$ BIPHEP system ${ }^{13}$ or the $\left[\mathrm{Ru}((R)-\mathrm{MeO}-\mathrm{BIPHEP}) \mathrm{Br}_{2}\right]$ complex $^{14}$ to furnish compounds $\mathbf{2 a - 2 d}$ in good yields (90-99\%) and with excellent enantiomeric excesses (95-99\% ee).<smiles>[R]C(=O)CC(=O)OCC</smiles>

1<smiles>[R]C(O)CC(=O)OCC</smiles>

2

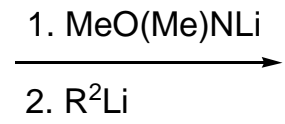<smiles>[R]C(=O)CC([R])O</smiles>

3

\section{Scheme 2}


Table 1. Preparation of hydroxy ketones $\mathbf{3 a - 3 g}$

Entry

${ }^{\mathrm{a}}$ Conditions for the asymmetric hydrogenation reactions: 2a: $0.3 \mathrm{~mol} \% \mathrm{RuCl}_{3} /(R)-\mathrm{MeO}-$ BIPHEP, $\mathrm{H}_{2}$ (10 bar), EtOH, $80^{\circ} \mathrm{C}, 24 \mathrm{~h} ; 2 \mathbf{b}: 1 \mathrm{~mol} \% \mathrm{RuCl}_{3} /(R)$-MeO-BIPHEP, $\mathrm{H}_{2}$ (20 bar), EtOH, $80^{\circ} \mathrm{C}$, 24h;. 2c: $1 \mathrm{~mol} \% \mathrm{RuCl}_{3} /(R)$-MeO-BIPHEP, $\mathrm{H}_{2}$ (30 bar), EtOH, $80^{\circ} \mathrm{C}, 48 \mathrm{~h}$; $2 \mathrm{~d}: 0.2$ $\mathrm{mol} \%\left[\mathrm{Ru}((R)-\mathrm{MeO}-\mathrm{BIPHEP}) \mathrm{Br}_{2}\right], \mathrm{H}_{2}(10 \mathrm{bar}), \mathrm{EtOH}, 80^{\circ} \mathrm{C}, 48 \mathrm{~h} .{ }^{\mathrm{b}}$ Enantiomeric excesses were determined by HPLC analysis (Chiralcel OD-H column, hexane $/ i \operatorname{PrOH}, \lambda=215$ or $254 \mathrm{~nm}$ ). ${ }^{\mathrm{c}}$ The absolute configurations of the hydroxy esters $\mathbf{2 a},{ }^{15} \mathbf{2} \mathbf{b},{ }^{16} \mathbf{2} \mathbf{c},{ }^{17} \mathbf{2 d}^{18}$ were assigned by comparison of their specific rotations with those reported in the literature.

These $\beta$-hydroxy esters $\mathbf{2}$ were then converted into various $\beta$-hydroxy ketones $\mathbf{3}$ following a two-step sequence (Scheme 2, Table 1). Reaction of 2a-2d with $N, O$-dimethylhydroxylamine hydrochloride (3 equiv.) and $n$-butyllithium (6 equiv.) afforded the corresponding Weinreb amides $^{19}$ and subsequent treatment with organolithium reagents (3 equiv.) delivered $\mathbf{3 a - 3} \mathbf{g}$ in good overall yields (60-93\%). Having synthesized a series of variously substituted enantiomerically pure $\beta$-hydroxy ketones, we were now able to study the ruthenium-mediated diastereoselective hydrogenation of these compounds using either MeO-BIPHEP ${ }^{20}$ or SYNPHOS $^{\mathbb{R} 21}$ as the chiral ligand. To our knowledge, only one example of ruthenium-mediated 
hydrogenation of a $\beta$-hydroxy ketone has been reported in the literature during studies on the hydrogenation of pentan-2,4-dione into the corresponding anti-1,3-diol. ${ }^{\text {a }}$ (2R)-Hydroxy-4pentanone, the intermediate isolated during the hydrogenation of pentan-2,4-dione, has been reduced with both $(R)$ and $(S)$-BINAP-Ru complexes into respectively the corresponding anti and syn-1,3-diols. We have thus undertaken a systematic study of the diastereoselective reduction of several diversely substituted $\beta$-hydroxy ketones (Scheme 3, Table 2).

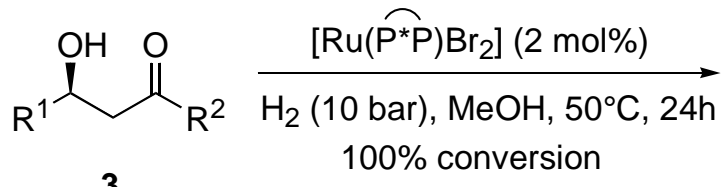

3<smiles>[R]C(O)CC([R])O</smiles>

$\overparen{P^{*} \mathrm{P}}=$

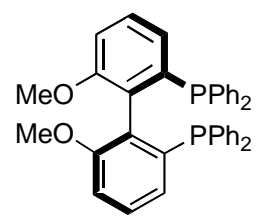

R)-MeO-BIPHEP

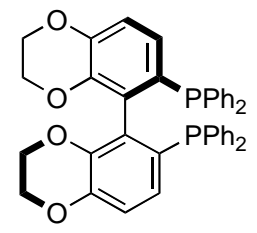

(R)-SYNPHOS

\section{Scheme 3}

Table 2. Diastereoselective hydrogenation of $\beta$-hydroxy ketones 3 using chiral ruthenium complexes $^{\mathrm{a}}$

Entry


Table 2. Continued

6<smiles>CCCCC(=O)C[C@@H](O)c1ccccc1</smiles>

7<smiles>CC(=O)CC(O)COCc1ccccc1</smiles>

3f

(R)-SYNPHOS<smiles>CCCC[C@@H](O)C[C@@H](O)c1ccccc1</smiles>

$(1 S, 3 R)-4 f$

$99^{d}$

$3 g$ (R)-MeO-BIPHEP<smiles>CC(O)CC(O)CO[Bi]</smiles>

$(2 S, 4 R)-4 g$

${ }^{a}$ The hydrogenation reaction can be performed at room temperature, but in this case a longer reaction time $(48 \mathrm{~h})$ was required to achieve full conversion with no incidence on the $d e .{ }^{\mathrm{b}} \mathrm{de}$ determined by GC analysis (DB1701 column) on the corresponding Mosher diesters. ${ }^{\mathrm{c}} d e$ determined by HPLC analysis: Chiralcel AS-H column, hexane/propan-2-ol. ${ }^{\mathrm{d}}$ de determined by HPLC analysis: Chiralcel OD-H column, hexane/propan-2-ol.

The hydrogenation reactions were first carried out with the atropisomeric diphosphine having the $(R)$ configuration in order to synthesize the corresponding anti-1,3-diols. Thus, all reactions were performed in methanol at $50^{\circ} \mathrm{C}$ under 10 bar of hydrogen with 2 mol\% of $\left[\mathrm{Ru}\left(\mathrm{P}^{*} \mathrm{P}\right) \mathrm{Br}_{2}\right]$ complex. In all cases the expected anti-1,3-diols were obtained quantitatively and with a high level of diastereoselectivity, ranging from $98 \%$ to $99.5 \%$ (Table 2 ).

It appears from these results that the nature of the $\mathrm{R}^{1}$ and $\mathrm{R}^{2}$ substituents on compounds 3 has no influence on the stereochemical outcome of the hydrogenation reaction and neither steric nor electronic effects have been observed since the anti diastereoselectivities were invariably high using either $(R)$-MeO-BIPHEP or $(R)$-SYNPHOS as a ligand.

For comparison, $\beta$-hydroxy ketones $\mathbf{3 b}$ and $\mathbf{3 f}$ have been reduced with tetramethylammonium triacetoxyborohydride, the most commonly used reagent for the diastereoselective reduction of this type of compounds. In both cases the diastereomeric excesses were high $(92 \%$ de $)$ but quite unsatisfactory compared to the diastereoselectivities obtained through ruthenium-mediated hydrogenation $(98-99.5 \%$ de) which stands for an efficient method for the preparation of anti1,3-diols.

We have then studied the hydrogenation of $\beta$-hydroxy ketone $\mathbf{3 b}$ using the ligand of opposite configuration, $(S)$-MeO-BIPHEP in order to achieve the corresponding 1,3-syn-diol (Scheme 4).

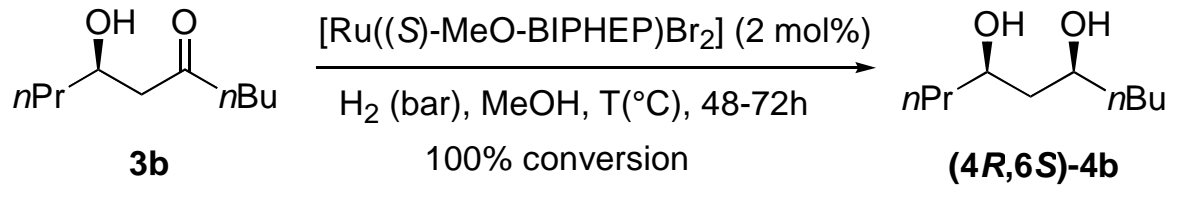

Scheme 4 
Table 3. Hydrogenation of $\beta$-hydroxy ketone 3b using [Ru((S)-MeO-BIPHEP)Br 2$]$

\begin{tabular}{cccc}
\hline Entry & $\mathrm{T}\left({ }^{\circ} \mathrm{C}\right)$ & $\mathrm{P}($ bar $)$ & de $(\%)$ \\
\hline 1 & 10 & 10 & 76 \\
2 & 25 & 10 & 72 \\
3 & 50 & 10 & 60 \\
4 & 25 & 5 & 69 \\
5 & 25 & 60 & 69 \\
6 & 80 & 60 & 46 \\
\hline
\end{tabular}

A short study of the influence of the temperature and the hydrogen pressure on the diastereoselectivity of the reaction has been carried out (Table 3). The hydrogenation was run in methanol using $2 \mathrm{~mol} \%$ of the $\left[\mathrm{Ru}((S)-\mathrm{MeO}-\mathrm{BIPHEP}) \mathrm{Br}_{2}\right]$ complex. A temperature effect has been observed since a decrease of diastereoselectivity (from 76 to $60 \% \mathrm{de}$ ) was noted when switching from 10 to $50^{\circ} \mathrm{C}$ (entries 1 to 3 ). On the other hand, the hydrogen pressure has no effect on the stereochemical outcome of the reaction since at $25^{\circ} \mathrm{C}$ identical diastereoselectivities $(69 \%$ de) were obtained at either 5 or 60 bar (entries 4 and 5$)$. At both higher temperature $\left(80^{\circ} \mathrm{C}\right)$ and hydrogen pressure (60 bar), lower diastereomeric excess was obtained ( $46 \%$ de, entry 6 ).

Likewise, hydrogenation of compounds 3a, 3c, 3g with $2 \mathrm{~mol} \%$ of $[\mathrm{Ru}((S)-\mathrm{MeO}-$ $\left.\mathrm{BIPHEP}) \mathrm{Br}_{2}\right]$ at $25^{\circ} \mathrm{C}$ under 10 bar of hydrogen proceeded with complete conversion and the expected syn-1,3-diols were obtained with only moderate diastereoselectivities ranging from $70 \%$ to $78 \%$ (Table 4 ).

Table 4. Hydrogenation of $\beta$-hydroxy ketones 3 using $\left[\mathrm{Ru}((S)-\mathrm{MeO}-\mathrm{BIPHEP}) \mathrm{Br}_{2}\right]^{\mathrm{a}}$

\begin{tabular}{cccc}
\hline Entry & Substrate 3 & $1,3-s y n$-Diol 4 & $d e(\%)$ \\
\hline 1 & 3a & $(\mathbf{2 S}, \mathbf{4 R})-\mathbf{4 a}$ & 70 \\
2 & $\mathbf{3 c}$ & $\mathbf{( 4 R , 6 S ) - 4 c}$ & 72 \\
3 & $\mathbf{3 g}$ & $(\mathbf{2 S}, \mathbf{4 S}) \mathbf{- 4 g}$ & 78 \\
\hline
\end{tabular}

${ }^{a}$ When the $(S)$-SYNPHOS ligand was used instead of $(S)$-MeO-BIPHEP, longer reaction times were required to achieve full conversion at $25^{\circ} \mathrm{C}$ whereas identical syn diastereoselectivities were obtained.

In conclusion, the ruthenium-mediated hydrogenation of $\beta$-hydroxy ketones exhibits both high levels of diastereoselection and a satisfactory degree of generality for the preparation of anti-1,3-diols. This catalytic method could be regarded as an interesting alternative to the reduction of $\beta$-hydroxy ketones with tetramethylammonium triacetoxyborohydride and should be particularly useful in total synthesis. As a synthetic application of this methodology, we have recently reported a formal synthesis of (-)-isoavenaciolide, ${ }^{22}$ a naturally occurring secondary metabolite isolated from the fermentation broth of Aspergillus and Penicillium species, and exhibiting antifungal activity. 


\section{Experimental Section}

General Procedures. All solvents were reagent grade and distilled under positive pressure of argon prior to use. Amines and $\mathrm{CH}_{2} \mathrm{Cl}_{2}$ were distilled from calcium hydride. THF and $\mathrm{Et}_{2} \mathrm{O}$ were distilled from sodium-benzophenone. Unless special mention, all reactions were carried out under an argon atmosphere. All commercially available reagents were used without further purification unless otherwise indicated. Nuclear magnetic resonance: ${ }^{1} \mathrm{H}-$ and ${ }^{13} \mathrm{C}-\mathrm{NMR}$ spectra were recorded either at $200 \mathrm{MHz}$ and $50 \mathrm{MHz}$ respectively on an AC200 Bruker spectrometer, or at $300 \mathrm{MHz}$ and $75 \mathrm{MHz}$ respectively on an Avance 300 Bruker spectrometer. Infrared spectra (IR) were recorded on either a Perkin-Elmer 783G spectrometer or an IRFT Nicolet 205 spectrometer. Mass spectra (MS) were measured on a Nermag R10-10C mass spectrometer $\left(\mathrm{DCI} / \mathrm{NH}_{3}\right)$ and on a PE Sciex API 3000 mass spectrometer (ESI). Flash column chromatography was performed on Merck silica gel (0.040-0.063 mesh). Thin layer chromatography (TLC) analysis was performed on Merck silica gel 60 PF 254 and revealed with either a ultra-violet lamp $(\lambda=254 \mathrm{~nm})$ or a potassium permanganate solution. Melting points (m.p.) were determined on a Kofler melting point apparatus and are uncorrected. Optical rotation values were recorded on a Perkin-Elmer 241 polarimeter at $589 \mathrm{~nm}$ (sodium lamp). High Performance Liquid Chromatography analyses (HPLC) were performed on a Waters instrument (Waters 486 detector, 717 autosampler equipped with Daicel Chiralcel OA, OB, OD, OD-H, OJ and Chiralpack AD and AS-H).

\section{Typical procedure for the catalytic hydrogenation of $\beta$-keto esters $1 \mathrm{a}-1 \mathrm{~d}$ with $\left[\mathrm{RuCl}_{3} /(\mathrm{R})\right.$ - MeO-BIPHEP]}

$(R)$-MeO-BIPHEP (0.01 equiv.) and anhydrous $\mathrm{RuCl}_{3}$ (0.01 equiv., purchased from Aldrich Chemicals) were placed in a round-bottomed tube and degassed by three vacuum/argon cycles at room temperature. $\beta$-Keto ester 1 ( 1 to 3.3 equiv.) was added followed by degassed methanol. The reaction vessel was placed in a stainless steel autoclave, which was purged with hydrogen and pressurized under 10-30 bar. The autoclave was heated to the desired temperature by circulating thermostated water in the double wall and magnetic stirring was started as soon as the required temperature was reached. After stirring for $24-48 \mathrm{~h}$, the autoclave was cooled to room temperature, hydrogen was vented and the reaction mixture was concentrated in vacuo and purified by flash chromatography. For $\beta$-keto ester 1d, the asymmetric reduction was run using the procedure described for hydrogenation of compounds 3. $\beta$-Hydroxy esters $\mathbf{2 a - 2 d}$ are known compounds, and the spectral data agreed with the literature reports. ${ }^{15-18}$

\section{Typical procedure for the preparation of $\beta$-hydroxy ketones 3a-3g from $\beta$-hydroxy esters 2a-2d}

To a solution of $\mathrm{N}, \mathrm{O}$-dimethylhydroxylamine hydrochloride $(6.69 \mathrm{~g}, 67.2 \mathrm{mmol})$ in THF (120 $\mathrm{mL}$ ) was added $n \mathrm{BuLi}(134 \mathrm{mmol})$ at $-78^{\circ} \mathrm{C}$. After stirring at room temperature for $10 \mathrm{~min}$, the mixture was cooled to $-78^{\circ} \mathrm{C}$ and a solution of $\beta$-hydroxy ester $2(22.4 \mathrm{mmol})$ in THF $(35 \mathrm{~mL})$ 
was added. The reaction mixture was stirred at $-78^{\circ} \mathrm{C}$ for $2 \mathrm{~h}$, then quenched with saturated aqueous $\mathrm{NH}_{4} \mathrm{Cl}$ and allowed to warm to room temperature. After extraction with $\mathrm{Et}_{2} \mathrm{O}$, the combined organic layers were dried over $\mathrm{MgSO}_{4}$ and concentrated. Purification of the residue by flash chromatography on silica gel (elution with cyclohexane/AcOEt: $1 / 1$ to 3/7) afforded the corresponding pure Weinreb amide $(21.7 \mathrm{mmol}, 97 \%)$. To a solution of this Weinreb amide $(9.6$ $\mathrm{mmol})$ in $\mathrm{THF}(20 \mathrm{~mL})$ at $-78^{\circ} \mathrm{C}$ was added dropwise the corresponding alkyl lithium $(28.7$ mmol). After stirring at $-78^{\circ} \mathrm{C}$ for $0.5 \mathrm{~h}$, the reaction mixture was quenched with methanol and saturated aqueous $\mathrm{NH}_{4} \mathrm{Cl}$, then allowed to warm to room temperature and extracted with $\mathrm{Et}_{2} \mathrm{O}$. The combined organic layers were dried over $\mathrm{MgSO}_{4}$ and concentrated. Purification of the residue by flash chromatography on silica gel (elution with cyclohexane/AcOEt: 8/2) afforded the pure $\beta$-hydroxy ketone $\mathbf{3}$.

(4R)-4-Hydroxyheptan-2-one (3a). 60\% yield (two steps), pale yellow oil; $\mathrm{R}_{\mathrm{f}}=0.10$ (cyclohexane/AcOEt 7/3, $\left.\mathrm{KMnO}_{4}\right) ;{ }^{1} \mathrm{H} \mathrm{NMR}\left(\mathrm{CDCl}_{3}, 200 \mathrm{MHz}\right): \delta 0.92(\mathrm{t}, J=6.6 \mathrm{~Hz}, 3 \mathrm{H}), 1.36$ $(\mathrm{m}, 4 \mathrm{H}), 2.18(\mathrm{~s}, 3 \mathrm{H}), 2.50(\mathrm{dd}, J=16.4$ and $8.4 \mathrm{~Hz}, 1 \mathrm{H}), 2.64(\mathrm{dd}, J=16.4$ and $3.5 \mathrm{~Hz}, 1 \mathrm{H})$, $4.04(\mathrm{~m}, 1 \mathrm{H}) ;{ }^{13} \mathrm{C} \mathrm{NMR}\left(\mathrm{CDCl}_{3}, 50 \mathrm{MHz}\right): \delta 14.0,18.7,30.8,38.6,50.1,67.3,210.1$; MS (DCI / $\left.\mathrm{NH}_{3}\right): \mathrm{m} / \mathrm{z}=132[\mathrm{M}+\mathrm{H}]^{+}, 148\left[\mathrm{M}+\mathrm{NH}_{4}\right]^{+}$; IR (thin film): $3426,2960,2925,2873,1701 \mathrm{~cm}^{-1}$; $[\alpha]_{\mathrm{D}}^{25}-49.6\left(c 0.23, \mathrm{CHCl}_{3}\right)$.

(7R)-7-Hydroxydecan-5-one (3b). 75\% yield (two steps), pale yellow oil; $\mathrm{R}_{\mathrm{f}}=0.65$ (cyclohexane/AcOEt 1/1, $\left.\mathrm{KMnO}_{4}\right) ;{ }^{1} \mathrm{H} \mathrm{NMR}\left(\mathrm{CDCl}_{3}, 200 \mathrm{MHz}\right): \delta 0.90(\mathrm{t}, J=7.2 \mathrm{~Hz}, 3 \mathrm{H}), 0.92$ $(\mathrm{t}, J=7.2 \mathrm{~Hz}, 3 \mathrm{H}), 1.43(\mathrm{~m}, 8 \mathrm{H}), 2.36(\mathrm{t}, J=7.6 \mathrm{~Hz}, 2 \mathrm{H}), 2.52(\mathrm{dd}, J=17.6$ and $8.6 \mathrm{~Hz}, 1 \mathrm{H})$, $2.60(\mathrm{dd}, J=17.6$ and $3.4 \mathrm{~Hz}, 1 \mathrm{H}), 4.03(\mathrm{~m}, 1 \mathrm{H}) ;{ }^{13} \mathrm{C} \mathrm{NMR}\left(\mathrm{CDCl}_{3}, 50 \mathrm{MHz}\right): \delta 13.8,14.0,18.7$, 22.3, 25.7, 38.7, 43.4, 49.1, 67.4, 212.5; MS (DCI / NH 3$): \mathrm{m} / \mathrm{z}=173[\mathrm{M}+\mathrm{H}]^{+}, 190\left[\mathrm{M}+\mathrm{NH}_{4}\right]^{+}$; IR (thin film): $3452,2966,2930,2870,1711 \mathrm{~cm}^{-1} ;[\alpha]_{\mathrm{D}}{ }^{25}-38.4\left(c 1.26, \mathrm{CHCl}_{3}\right)$.

(4R)-4-Hydroxytetradecan-6-one (3c). 75\% yield (two steps), pale yellow oil; $\mathrm{R}_{\mathrm{f}}=0.63$ (cyclohexane/AcOEt 1/1, $\left.\mathrm{KMnO}_{4}\right) ;{ }^{1} \mathrm{H} \mathrm{NMR}\left(\mathrm{CDCl}_{3}, 200 \mathrm{MHz}\right): \delta 0.90(\mathrm{t}, J=7.2 \mathrm{~Hz}, 3 \mathrm{H}), 0.95$ $(\mathrm{t}, J=7.2 \mathrm{~Hz}, 3 \mathrm{H}), 1.25$ (br s, 10H), $1.50(\mathrm{~m}, 4 \mathrm{H}), 2.41(\mathrm{t}, J=7.4 \mathrm{~Hz}, 2 \mathrm{H}), 2.46$ (dd, $J=16.4$ and $8.4 \mathrm{~Hz}, 1 \mathrm{H}), 2.60(\mathrm{dd}, J=16.4$ and $3.7 \mathrm{~Hz}, 1 \mathrm{H}), 4.05(\mathrm{~m}, 1 \mathrm{H}) ;{ }^{13} \mathrm{C} \mathrm{NMR}\left(\mathrm{CDCl}_{3}, 50 \mathrm{MHz}\right): \delta$ 13.9, 14.0, 18.6, 22.6, 23.6, 29.1, 29.2, 29.3, 31.8, 38.6, 43.7, 48.9, 67.3, 212.6; MS (DCI / NH$)$ : $\mathrm{m} / \mathrm{z}=229[\mathrm{M}+\mathrm{H}]^{+}, 246\left[\mathrm{M}+\mathrm{NH}_{4}\right]^{+}$; IR (thin film): 3518, 2966, 2950, 2827, $1705 \mathrm{~cm}^{-1} ;[\alpha]_{\mathrm{D}}{ }^{25}{ }_{-}$ 28.7 (c 1.10, $\left.\mathrm{CHCl}_{3}\right)$.

(6S)-6-Hydroxy-7-methyl-octan-4-one (3d). 69\% yield (two steps), pale yellow oil; $\mathrm{R}_{\mathrm{f}}=0.22$ (petroleum ether/ $\left.\mathrm{Et}_{2} \mathrm{O} 7 / 3, \mathrm{KMnO}_{4}\right) ;{ }^{1} \mathrm{H} \mathrm{NMR}\left(\mathrm{CDCl}_{3}, 300 \mathrm{MHz}\right): \delta 0.91(\mathrm{~d}, J=6.8 \mathrm{~Hz}, 3 \mathrm{H})$, 0.92 (t, $J=7.4 \mathrm{~Hz}, 3 \mathrm{H}), 0.93(\mathrm{~d}, J=6.8 \mathrm{~Hz}, 3 \mathrm{H}), 1.61$ (hex, $J=7.4 \mathrm{~Hz}, 2 \mathrm{H}), 1.68(\mathrm{~m}, 1 \mathrm{H}), 2.42$ (t, $J=7.4 \mathrm{~Hz}, 2 \mathrm{H}), 2.47(\mathrm{dd}, J=17.3$ and $9.3 \mathrm{~Hz}, 1 \mathrm{H}), 2.58(\mathrm{dd}, J=17.3$ and $2.8 \mathrm{~Hz}, 1 \mathrm{H}), 3.80$ (ddd, $J=9.3,2.8$ and $5.9 \mathrm{~Hz}, 1 \mathrm{H}) ;{ }^{13} \mathrm{C} \mathrm{NMR}\left(\mathrm{CDCl}_{3}, 75 \mathrm{MHz}\right): \delta 13.6,17.0,17.7,18.3,32.9$, 45.5, 45.8, 72.2, 212.7; MS (DCI / NH $): \mathrm{m} / \mathrm{z}=141\left[\mathrm{M}-\mathrm{H}_{2} \mathrm{O}+\mathrm{H}\right]^{+}, 159[\mathrm{M}+\mathrm{H}]^{+}, 176\left[\mathrm{M}+\mathrm{NH}_{4}\right]^{+}$; IR (thin film): 3440, 2970, 2940, 2880, $1715 \mathrm{~cm}^{-1}$; $[\alpha]_{\mathrm{D}}{ }^{25}-61.7\left(c 1.60, \mathrm{CHCl}_{3}\right)$.

(3S)-3-Hydroxy-4-methyl-1-phenyl-pentan-1-one (3e). 62\% yield (two steps), pale yellow oil; $\mathrm{R}_{\mathrm{f}}=0.55$ (cyclohexane/AcOEt 6/4, $\left.\mathrm{KMnO}_{4}, \mathrm{UV}\right) ;{ }^{1} \mathrm{H} \mathrm{NMR}\left(\mathrm{CDCl}_{3}, 300 \mathrm{MHz}\right): \delta 0.99(\mathrm{~d}, J=$ $6.8 \mathrm{~Hz}, 3 \mathrm{H}), 1.02(\mathrm{~d}, J=6.8 \mathrm{~Hz}, 3 \mathrm{H}), 1.81(\mathrm{~m}, 1 \mathrm{H}), 3.03(\mathrm{dd}, J=17.5$ and $9.4 \mathrm{~Hz}, 1 \mathrm{H}), 3.18(\mathrm{dd}$, $J=17.5$ and $2.5 \mathrm{~Hz}, 1 \mathrm{H}), 4.00$ (ddd, $J=9.4,5.6$ and $2.5 \mathrm{~Hz}, 1 \mathrm{H}), 7.40(\mathrm{~m}, 2 \mathrm{H}), 7.56(\mathrm{~m}, 1 \mathrm{H})$, $7.95(\mathrm{~m}, 2 \mathrm{H}) ;{ }^{13} \mathrm{C} \mathrm{NMR}\left(\mathrm{CDCl}_{3}, 75 \mathrm{MHz}\right): \delta 18.0,18.6,33.2,42.1,72.5,128.2,128.7,133.5$, 
137.1, 201.4; MS (DCI / $\left.\mathrm{NH}_{3}\right): \mathrm{m} / \mathrm{z}=193[\mathrm{M}+\mathrm{H}]^{+}, 210\left[\mathrm{M}+\mathrm{NH}_{4}\right]^{+}$; IR (thin film): 3470, 3070, 2970, 2940, 2880, 1680, 755, $690 \mathrm{~cm}^{-1} ;[\alpha]_{\mathrm{D}}^{25}-83.0\left(c 0.50, \mathrm{CHCl}_{3}\right)$.

(1S)-1-Hydroxy-1-phenylheptan-3-one (3f). 93\% yield (two steps), pale yellow oil; $\mathrm{R}_{\mathrm{f}}=0.31$ (cyclohexane/AcOEt 7/3, $\left.\mathrm{KMnO}_{4}, \mathrm{UV}\right) ;{ }^{1} \mathrm{H} \mathrm{NMR}\left(\mathrm{CDCl}_{3}, 300 \mathrm{MHz}\right): \delta 0.90(\mathrm{t}, J=7.2 \mathrm{~Hz}, 3 \mathrm{H})$, 1.31 (hex, $J=7.4 \mathrm{~Hz}, 2 \mathrm{H}$ ), 1.57 (qn, $J=7.5 \mathrm{~Hz}, 2 \mathrm{H}), 2.44$ (t, $J=7.3 \mathrm{~Hz}, 2 \mathrm{H}), 2.78$ (dd, $J=17.2$ and $4.1 \mathrm{~Hz}, 1 \mathrm{H}), 2.86(\mathrm{dd}, J=17.2$ and $8.3 \mathrm{~Hz}, 1 \mathrm{H}), 5.16(\mathrm{dt}, J=8.3$ and $3.6 \mathrm{~Hz}, 1 \mathrm{H}), 7.25-7.40$ $(\mathrm{m}, 5 \mathrm{H}) ;{ }^{13} \mathrm{C} \mathrm{NMR}\left(\mathrm{CDCl}_{3}, 75 \mathrm{MHz}\right): \delta 13.8,22.2,25.6,43.4,51.0,69.9,125.6,127.6,128.5$, 142.9, 211.6; MS (DCI / NH $): \mathrm{m} / \mathrm{z}=189\left[\mathrm{M}-\mathrm{H}_{2} \mathrm{O}+\mathrm{H}\right]^{+}, 206[\mathrm{M}+\mathrm{H}]^{+}, 224\left[\mathrm{M}+\mathrm{NH}_{4}\right]^{+}$; IR (thin film): 3430, 3070, 3040, 2970, 2940, 1730, 760, $700 \mathrm{~cm}^{-1}$; $[\alpha]_{\mathrm{D}}{ }^{25}-61.6\left(c 1.00, \mathrm{CHCl}_{3}\right)$.

(4S)-5-(Benzyloxy)-4-hydroxypentan-2-one (3g). 65\% yield (two steps), colorless oil; $\mathrm{R}_{\mathrm{f}}=0.64$ (cyclohexane/AcOEt 6/4, UV and $\left.\mathrm{KMnO}_{4}\right) ;{ }^{1} \mathrm{H} \mathrm{NMR}\left(\mathrm{CDCl}_{3}, 200 \mathrm{MHz}\right): \delta 2.18(\mathrm{~s}, 3 \mathrm{H}), 2.61$ (dd, $J=17.4$ and $5.2 \mathrm{~Hz}, 1 \mathrm{H}), 2.71(\mathrm{dd}, J=17.4$ and $6.6 \mathrm{~Hz}, 1 \mathrm{H}), 3.43(\mathrm{dd}, J=9.7$ and $5.8 \mathrm{~Hz}$, $1 \mathrm{H}), 3.49(\mathrm{dd}, J=9.7$ and $4.7 \mathrm{~Hz}, 1 \mathrm{H}), 4.26(\mathrm{~m}, 1 \mathrm{H}), 4.52(\mathrm{~d}, J=12.0 \mathrm{~Hz}, 1 \mathrm{H}), 4.58(\mathrm{~d}, J=12.0$ $\mathrm{Hz}, 1 \mathrm{H}), 7.25-7.3(\mathrm{~m}, 5 \mathrm{H}) ;{ }^{13} \mathrm{C} \mathrm{NMR}\left(\mathrm{CDCl}_{3}, 50 \mathrm{MHz}\right): \delta 30.9,46.7,66.9,73.3,73.5,127.8$, 127.9, 128.5, 138.0, 209.7; MS (DCI / NH 3$): \mathrm{m} / \mathrm{z}=209[\mathrm{M}+\mathrm{H}]^{+}, 226\left[\mathrm{M}+\mathrm{NH}_{4}\right]^{+}$; IR (thin film): $3452,3063,3032,2909,2863,1721 \mathrm{~cm}^{-1} ;[\alpha]_{\mathrm{D}}{ }^{25}-15.5\left(c 1.36, \mathrm{CHCl}_{3}\right)$.

Typical procedure for the catalytic hydrogenation of $\beta$-hydroxy ketones 3a-3g with [Ru((R)-MeO-BIPHEP)Br 2$]$ or $\left[\operatorname{Ru}((R)-S Y N P H O S) B r_{2}\right]$

Either $(R)$-MeO-BIPHEP $(7.0 \mathrm{mg}, 0.012 \mathrm{mmol})$ or $(R)-S Y N P H O S(7.7 \mathrm{mg}, 0.012 \mathrm{mmol})$ and (COD)Ru(2-methylallyl) 2 (3.2 mg, $0.01 \mathrm{mmol}$, commercially available from Acros) were placed in a round-bottomed tube, degassed by three vacuum/argon cycles at room temperature, and dissolved in degassed acetone $(1 \mathrm{~mL})$. To this suspension was added at room temperature a 0.15 $\mathrm{N}$ methanolic hydrobromic acid solution $(147 \mu \mathrm{L}, 0.022 \mathrm{mmol})$ and the mixture was stirred at $25^{\circ} \mathrm{C}$ for $30 \mathrm{~min}$. After evaporation of the solvent under vacuum, a solution of $\beta$-hydroxy ketone $3(0.5 \mathrm{mmol})$ in $\mathrm{MeOH}(1 \mathrm{~mL})$ was added. The reaction vessel was placed in a stainless steel autoclave which was purged with hydrogen and pressurized under 10 bar. The autoclave was heated to $50^{\circ} \mathrm{C}$ by circulating thermostated water in the double wall and magnetic stirring was started as soon as the required temperature was reached. After stirring for $24 \mathrm{~h}$, the autoclave was cooled to room temperature, hydrogen was vented and the reaction mixture was concentrated in vacuo. ${ }^{1} \mathrm{H}$ NMR of the crude product showed that full conversion was achieved. Purification of the residue by flash chromatography afforded pure anti-1,3-diol 4. The syn-1,3-diols were prepared using $(S)$-MeO-BIPHEP as a ligand.

(2R,4R)-Heptane-2,4-diol [(2R,4R)-4a]. $\mathrm{R}_{\mathrm{f}}=0.43$ (cyclohexane/AcOEt $\left.1 / 1, \mathrm{KMnO}_{4}\right) ;{ }^{1} \mathrm{H} \mathrm{NMR}$ $\left(\mathrm{CDCl}_{3}, 200 \mathrm{MHz}\right): \delta 0.90(\mathrm{t}, J=6.8 \mathrm{~Hz}, 3 \mathrm{H}), 1.20(\mathrm{~d}, J=6.4 \mathrm{~Hz}, 3 \mathrm{H}), 1.40(\mathrm{~m}, 4 \mathrm{H}), 1.56(\mathrm{~m}$, $2 \mathrm{H}), 3.92(\mathrm{~m}, 1 \mathrm{H}), 4.13(\mathrm{sext}, J=6.0 \mathrm{~Hz}, 1 \mathrm{H}) ;{ }^{13} \mathrm{C} \mathrm{NMR}\left(\mathrm{CDCl}_{3}, 50 \mathrm{MHz}\right): \delta 14.0,18.9,23.5$, 39.5, 40.0, 65.4, 69.0; MS (DCI / NH$): ~ m / z=133[\mathrm{M}+\mathrm{H}]^{+}, 150\left[\mathrm{M}+\mathrm{NH}_{4}\right]^{+}$; IR (thin film): 3398, 2969, 2930, $2875 \mathrm{~cm}^{-1}$; $[\alpha]_{\mathrm{D}}{ }^{25}-21.7$ (c 1.15, $\mathrm{CHCl}_{3}$ ); $\mathrm{GC}$ analysis (diester with $(S)$-Mosher chloride): Column, J\&W Scientific DB1701; gas, helium; flow: $1 \mathrm{~mL} / \mathrm{min} ; \mathrm{T}_{\text {injector }}: 250^{\circ} \mathrm{C}$; $\mathrm{T}_{\text {detector }}: 260^{\circ} \mathrm{C}$; $\mathrm{T}_{\text {oven }}: 210^{\circ} \mathrm{C}(1 \mathrm{~min})$ then $10^{\circ} \mathrm{C} / \mathrm{min}$ to $250^{\circ} \mathrm{C} ; t_{R(2 R, 4 R)}=9.94 \mathrm{~min}, t_{R(2 S, 4 R)}=11.19$ $\min ; d e=98 \%$.

(2S,4R)-Heptane-2,4-diol [(2S,4R)-4a]. $\mathrm{R}_{\mathrm{f}}=0.47$ (cyclohexane/AcOEt 1/1, $\left.\mathrm{KMnO}_{4}\right)$; ${ }^{1} \mathrm{H}$ NMR $\left(\mathrm{CDCl}_{3}, 200 \mathrm{MHz}\right): \delta 0.90(\mathrm{t}, J=7.0 \mathrm{~Hz}, 3 \mathrm{H}), 1.20(\mathrm{~d}, J=6.2 \mathrm{~Hz}, 3 \mathrm{H}), 1.40(\mathrm{~m}, 6 \mathrm{H}), 3.83(\mathrm{~m}$, 
1H), $4.03(\mathrm{~m}, 1 \mathrm{H}) ;{ }^{13} \mathrm{C} \mathrm{NMR}\left(\mathrm{CDCl}_{3}, 50 \mathrm{MHz}\right): \delta 14.0,18.5,24.1,40.3,44.5,69.1,72.7$; $\mathrm{MS}$ (DCI / NH$\left.)_{3}\right): \mathrm{m} / \mathrm{z}=133[\mathrm{M}+\mathrm{H}]^{+}, 150\left[\mathrm{M}+\mathrm{NH}_{4}\right]^{+}$; IR (thin film): $3398,2969,2930,2875 \mathrm{~cm}^{-1}$

(4R,6R)-Decane-4,6-diol $[(\mathbf{4 R}, \mathbf{6 R})-4 b] . \mathrm{R}_{\mathrm{f}}=0.46$ (cyclohexane/AcOEt $\left.1 / 1, \mathrm{KMnO}_{4}\right)$; ${ }^{1} \mathrm{H}$ NMR $\left(\mathrm{CDCl}_{3}, 200 \mathrm{MHz}\right): \delta 0.89(\mathrm{t}, J=6.5 \mathrm{~Hz}, 3 \mathrm{H}), 0.92(\mathrm{t}, J=6.8 \mathrm{~Hz}, 3 \mathrm{H}), 1.39(\mathrm{~m}, 10 \mathrm{H}), 1.58(\mathrm{dd}$, $J=5.3$ and $6.2 \mathrm{~Hz}, 2 \mathrm{H}), 3.92(\mathrm{~m}, 2 \mathrm{H}) ;{ }^{13} \mathrm{C} \mathrm{NMR}\left(\mathrm{CDCl}_{3}, 50 \mathrm{MHz}\right): \delta 14.0,18.9,22.7,28.0$, 37.2, 39.6, 42.3, 69.1, 69.4; MS (DCI / $\left.\mathrm{NH}_{3}\right): \mathrm{m} / \mathrm{z}=175[\mathrm{M}+\mathrm{H}]^{+}, 192\left[\mathrm{M}+\mathrm{NH}_{4}\right]^{+}$; IR (thin film): $3413,2959,2935,2875 \mathrm{~cm}^{-1} ;[\alpha]_{\mathrm{D}}{ }^{25}-11.5\left(c 0.99, \mathrm{CHCl}_{3}\right)$; $\mathrm{GC}$ analysis (diester with $(S)$-Mosher chloride): Column, J\&W Scientific DB1701; gas, helium; flow: $1 \mathrm{~mL} / \mathrm{min} ; \mathrm{T}_{\text {injector: }} 250^{\circ} \mathrm{C}$; $\mathrm{T}_{\text {detector: }}: 260^{\circ} \mathrm{C} ; \mathrm{T}_{\text {oven }}: 210^{\circ} \mathrm{C}(1 \mathrm{~min})$ then $10^{\circ} \mathrm{C} / \mathrm{min}$ to $250^{\circ} \mathrm{C} ; t_{R(4 R, 6 R)}=13.29 \mathrm{~min}, t_{R(4 R, 6 S)}=$ $14.32 \mathrm{~min} ; \mathrm{de}=98 \%$.

(4R,6S)-Decane-4,6-diol $[(\mathbf{4 R}, \mathbf{6 S})-\mathbf{4 b}] . \mathrm{R}_{\mathrm{f}}=0.48$ (cyclohexane/AcOEt $\left.1 / 1, \mathrm{KMnO}_{4}\right)$; ${ }^{1} \mathrm{H} \mathrm{NMR}$ $\left(\mathrm{CDCl}_{3}, 200 \mathrm{MHz}\right): \delta 0.89(\mathrm{t}, J=6.5 \mathrm{~Hz}, 3 \mathrm{H}), 0.91(\mathrm{t}, J=6.8 \mathrm{~Hz}, 3 \mathrm{H}), 1.45(\mathrm{~m}, 12 \mathrm{H}), 3.83(\mathrm{~m}$, $2 \mathrm{H}) ;{ }^{13} \mathrm{C} \mathrm{NMR}\left(\mathrm{CDCl}_{3}, 50 \mathrm{MHz}\right): \delta 14.0,18.5,22.6,27.5,37.9,40.4,42.3,72.9,73.2$; MS (DCI $\left./ \mathrm{NH}_{3}\right): \mathrm{m} / \mathrm{z}=175[\mathrm{M}+\mathrm{H}]^{+}, 192\left[\mathrm{M}+\mathrm{NH}_{4}\right]^{+}$; IR (thin film): 3413, 2959, 2935, $2875 \mathrm{~cm}^{-1}$

(4R,6R)-Tetradecane-4,6-diol $[(\mathbf{4 R}, \mathbf{6 R})-\mathbf{4 c}] . \mathrm{R}_{\mathrm{f}}=0.49$ (cyclohexane/AcOEt 1/1, $\left.\mathrm{KMnO}_{4}\right) ;{ }^{1} \mathrm{H}$ NMR $\left(\mathrm{CDCl}_{3}, 200 \mathrm{MHz}\right): \delta 0.87(\mathrm{t}, J=6.6 \mathrm{~Hz}, 3 \mathrm{H}), 0.93(\mathrm{t}, J=6.6 \mathrm{~Hz}, 3 \mathrm{H}), 1.26($ br s, $10 \mathrm{H})$, $1.43(\mathrm{~m}, 8 \mathrm{H}), 1.58(\mathrm{dd}, J=6.0$ and $5.1 \mathrm{~Hz}, 2 \mathrm{H}), 3.92(\mathrm{~m}, 2 \mathrm{H}) ;{ }^{13} \mathrm{C} \mathrm{NMR}\left(\mathrm{CDCl}_{3}, 50 \mathrm{MHz}\right)$ : $\delta 14.1,18.9,22.6,25.8,29.2,29.5,29.6,31.8,37.5,39.6,42.3,69.1,69.4 ; \mathrm{MS}\left(\mathrm{DCI} / \mathrm{NH}_{3}\right)$ : $\mathrm{m} / \mathrm{z}=231[\mathrm{M}+\mathrm{H}]^{+}, 248\left[\mathrm{M}+\mathrm{NH}_{4}\right]^{+}$; IR (thin film): 3437, 2984, 2954, $2830 \mathrm{~cm}^{-1} ;[\alpha]_{\mathrm{D}}{ }^{25}-12.0(c$ 1.11, $\mathrm{CHCl}_{3}$ ); GC analysis (diester with $(S)$-Mosher chloride): Column, J\&W Scientific DB1701; gas, helium; flow: $1 \mathrm{~mL} / \mathrm{min}$; $\mathrm{T}_{\text {injector: }} 250^{\circ} \mathrm{C}$; $\mathrm{T}_{\text {detector }}: 260^{\circ} \mathrm{C}$; $\mathrm{T}_{\text {oven }}: 210^{\circ} \mathrm{C}(1 \mathrm{~min})$ then $2{ }^{\circ} \mathrm{C} / \mathrm{min}$ to $250^{\circ} \mathrm{C} ; t_{R(4 R, 6 R)}=27.52 \mathrm{~min}, t_{R(4 R, 6 S)}=30.22 \mathrm{~min} ; d e=99 \%$.

(4R,6S)-Tetradecane-4,6-diol $\left[\left(\mathbf{4 R , 6 S ) - 4 c ] . ~} \mathrm{R}_{\mathrm{f}}=0.51\right.\right.$ (cyclohexane/AcOEt 1/1, $\left.\mathrm{KMnO}_{4}\right) ;{ }^{1} \mathrm{H}$ NMR $\left(\mathrm{CDCl}_{3}, 200 \mathrm{MHz}\right): \delta 0.87(\mathrm{t}, J=6.6 \mathrm{~Hz}, 3 \mathrm{H}), 0.93(\mathrm{t}, J=6.6 \mathrm{~Hz}, 3 \mathrm{H}), 1.26($ br s, $10 \mathrm{H})$, $1.43(\mathrm{~m}, 8 \mathrm{H}), 1.58(\mathrm{dd}, J=6.0$ and $5.1 \mathrm{~Hz}, 2 \mathrm{H}), 3.92(\mathrm{~m}, 2 \mathrm{H}) ;{ }^{13} \mathrm{C} \mathrm{NMR}\left(\mathrm{CDCl}_{3}, 50 \mathrm{MHz}\right): \delta$ 14.0, 18.4, 22.5, 26.8, 29.2, 29.5, 29.6, 31.7, 38.2, 40.3, 42.7, 72.6, 72.9; $\mathrm{MS}\left(\mathrm{DCI} / \mathrm{NH}_{3}\right): \mathrm{m} / \mathrm{z}=$ $231[\mathrm{M}+\mathrm{H}]^{+}, 248\left[\mathrm{M}+\mathrm{NH}_{4}\right]^{+}$; IR (thin film): 3437, 2984, 2954, $2830 \mathrm{~cm}^{-1}$.

(3S,5R)-2-Methyloctane-3,5-diol [(3S,5R)-4d]. $\mathrm{R}_{\mathrm{f}}=0.44$ (cyclohexane/AcOEt 1/1, $\mathrm{KMnO}_{4}$ ); ${ }^{1} \mathrm{H}$ NMR $\left(\mathrm{CDCl}_{3}, 300 \mathrm{MHz}\right): \delta 0.89(\mathrm{~d}, J=6.9 \mathrm{~Hz}, 3 \mathrm{H}), 0.94(\mathrm{~d}, J=6.9 \mathrm{~Hz}, 3 \mathrm{H}), 1.50(\mathrm{~m}, 7 \mathrm{H})$, 3.66 (ddd, $J=3.0,6.2$ and $9.0 \mathrm{~Hz}, 1 \mathrm{H}), 3.94(\mathrm{~m}, 1 \mathrm{H}) ;{ }^{13} \mathrm{C} \mathrm{NMR}\left(\mathrm{CDCl}_{3}, 75 \mathrm{MHz}\right): \delta 14.1,18.1$, 18.7, 19.1, 33.8, 39.5, 39.6, 69.2, 73.9; IR (thin film): 3420, 2970, $2940 \mathrm{~cm}^{-1} ;[\alpha]_{\mathrm{D}}{ }^{25}-22.6(c$ $0.50, \mathrm{CHCl}_{3}$ ); GC analysis (diester with $(S)$-Mosher chloride): Column, J\&W Scientific DB1701; gas, helium; flow: $1 \mathrm{~mL} / \mathrm{min}$; $\mathrm{T}_{\text {injector: }}: 250^{\circ} \mathrm{C}$; $\mathrm{T}_{\text {detector }}: 260^{\circ} \mathrm{C}$; $\mathrm{T}_{\text {oven }}: 210^{\circ} \mathrm{C}(1 \mathrm{~min})$ then $5^{\circ} \mathrm{C} / \mathrm{min}$ to $250^{\circ} \mathrm{C} ; t_{R(3 S, 5 R)}=12.63 \mathrm{~min}, t_{R(3 S, 5 S)}=13.29 \mathrm{~min} ; \mathrm{de}=99 \%$.

(1S,3S)-4-Methyl-1-phenylpentane-1,3-diol [(1S,3S)-4e]. $\mathrm{R}_{\mathrm{f}}=0.19$ (cyclohexane/AcOEt $\left.75 / 25, \mathrm{KMnO}_{4}, \mathrm{UV}\right) ;{ }^{1} \mathrm{H} \mathrm{NMR}\left(\mathrm{CDCl}_{3}, 300 \mathrm{MHz}\right): \delta 0.88(\mathrm{~d}, J=6.8 \mathrm{~Hz}, 3 \mathrm{H}), 0.91(\mathrm{~d}, J=6.8$ $\mathrm{Hz}, 3 \mathrm{H}), 1.68$ (oct, $J=6.5 \mathrm{~Hz}, 1 \mathrm{H}), 1.86(\mathrm{~m}, 2 \mathrm{H}), 3.59(\mathrm{dt}, J=6.0$ and $5.8 \mathrm{~Hz}, 1 \mathrm{H}), 5.04(\mathrm{dd}, J=$ 5.0 and $6.5 \mathrm{~Hz}, 1 \mathrm{H}), 7.20-7.36(\mathrm{~m}, 5 \mathrm{H}) ;{ }^{13} \mathrm{C} \mathrm{NMR}\left(\mathrm{CDCl}_{3}, 75 \mathrm{MHz}\right): \delta 17.8,18.6,33.8,41.8$, 71.8, 73.9, 125.6, 127.3, 128.5,144.8; IR (thin film): 3450, 3070, 3040, 2970, $2945 \mathrm{~cm}^{-1}$; $[\alpha]_{\mathrm{D}}{ }^{25}{ }_{-}$ 73.5 ( c 0.50, $\mathrm{CHCl}_{3}$ ); HPLC analysis: Column, Chiralcel AS-H; eluent, hexane/propan-2-ol 95/5; flow rate: $1.0 \mathrm{~mL} / \mathrm{min}$; detection: $215 \mathrm{~nm} ; t_{R(1 R, 3 S)}=11.11 \mathrm{~min}, t_{R(1 S, 3 S)}=12.14 \mathrm{~min} ; \mathrm{de}=98 \%$.

(1S,3R)-1-Phenylheptane-1,3-diol $[(\mathbf{1 S}, \mathbf{3 R})-\mathbf{4 f}] \cdot \mathrm{R}_{\mathrm{f}}=0.41$ (cyclohexane/AcOEt 1/1, $\mathrm{KMnO}_{4}$, $\mathrm{UV}) ;{ }^{1} \mathrm{H}$ NMR $\left(\mathrm{CDCl}_{3}, 300 \mathrm{MHz}\right): \delta 0.89(\mathrm{t}, J=7.0 \mathrm{~Hz}, 3 \mathrm{H}), 1.31(\mathrm{~m}, 4 \mathrm{H}), 1.51(\mathrm{~m}, 2 \mathrm{H}), 1.84$ (ddd, $J=3.4,7.9$ and $11.5 \mathrm{~Hz}, 1 \mathrm{H}), 1.91(\mathrm{ddd}, J=3.4,7.9$ and $11.5 \mathrm{~Hz}, 1 \mathrm{H}), 3.86(\mathrm{~m}, 1 \mathrm{H}), 5.06$ $(\mathrm{dd}, J=3.4$ and $7.9 \mathrm{~Hz}, 1 \mathrm{H}), 7.25-7.35(\mathrm{~m}, 5 \mathrm{H}) ;{ }^{13} \mathrm{C} \mathrm{NMR}\left(\mathrm{CDCl}_{3}, 75 \mathrm{MHz}\right): \delta 14.1,22.8,27.9$, 
37.2, 44.6, 69.4, 71.8, 125.6, 127.4, 128.5, 144.7; MS (DCI / $\left.\mathrm{NH}_{3}\right): \mathrm{m} / \mathrm{z}=209[\mathrm{M}+\mathrm{H}]^{+}, 226$ $\left[\mathrm{M}+\mathrm{NH}_{4}\right]^{+}$; IR (thin film): 3390, 3070, 3040, 2960, 2935, 2870, 750, $700 \mathrm{~cm}^{-1 ;}[\alpha]_{\mathrm{D}}^{25}-42.9(c$ 0.25, $\mathrm{CHCl}_{3}$ ); HPLC analysis: Column, Chiralcel OD-H; eluent, hexane/propan-2-ol 95/5; flow rate: $1.0 \mathrm{~mL} / \mathrm{min}$; detection: $215 \mathrm{~nm} ; t_{R(I S, 3 R)}=12.21 \mathrm{~min}, t_{R(I S, 3 S)}=16.01 \mathrm{~min} ; d e=99 \%$.

(2S,4R)-1-(Benzyloxy)pentane-2,4-diol $[(\mathbf{2 S}, \mathbf{4 R})-\mathbf{4 g}] \cdot \mathrm{R}_{\mathrm{f}}=0.25$ (cyclohexane/AcOEt 1/1, $\left.\mathrm{KMnO}_{4}, \mathrm{UV}\right) ;{ }^{1} \mathrm{H}$ NMR $\left(\mathrm{CDCl}_{3}, 200 \mathrm{MHz}\right): \delta 1.19(\mathrm{~d}, J=6.4 \mathrm{~Hz}, 3 \mathrm{H}), 1.55(\mathrm{~m}, 2 \mathrm{H}), 3.39(\mathrm{dd}, J$ $=9.5$ and $7.3 \mathrm{~Hz}, 1 \mathrm{H}), 3.48(\mathrm{dd}, J=9.5$ and $4.0 \mathrm{~Hz}, 1 \mathrm{H}), 4.10(\mathrm{~m}, 2 \mathrm{H}), 4.55(\mathrm{~s}, 2 \mathrm{H}), 7.31(\mathrm{~m}$, $5 \mathrm{H}) ;{ }^{13} \mathrm{C} \mathrm{NMR}\left(\mathrm{CDCl}_{3}, 50 \mathrm{MHz}\right): \delta 23.6,40.8,64.9,67.9,73.3,74.5,127.7,127.8,128.5,137.8$; MS (DCI $\left./ \mathrm{NH}_{3}\right): \mathrm{m} / \mathrm{z}=211[\mathrm{M}+\mathrm{H}]^{+}, 228\left[\mathrm{M}+\mathrm{NH}_{4}\right]^{+}$; IR (thin film): 3410, 3055, 3030, 2980, 2930, 2860, 735, $700 \mathrm{~cm}^{-1}$; $[\alpha]_{\mathrm{D}}^{25}-10.4$ (c 1.17, $\left.\mathrm{CHCl}_{3}\right)$; HPLC analysis: Column, Chiralcel OD$\mathrm{H}$; eluent, hexane/propan-2-ol 90/10; flow rate: $1.0 \mathrm{~mL} / \mathrm{min}$; detection: $254 \mathrm{~nm} ; t_{R(2 S, 4 R)}=12.36$ $\min , t_{R(2 S, 4 S)}=19.93 \mathrm{~min} ; d e=99 \%$.

$(\mathbf{2 S}, \mathbf{4 S})-\mathbf{1 - ( B e n z y l o x y})$ pentane-2,4-diol $\quad[(\mathbf{2 S}, \mathbf{4 S})-\mathbf{4 g}] . \quad \mathrm{R}_{\mathrm{f}}=0.30 \quad$ (cyclohexane/AcOEt 1/1, $\left.\mathrm{KMnO}_{4}, \mathrm{UV}\right) ;{ }^{1} \mathrm{H}$ NMR $\left(\mathrm{CDCl}_{3}, 200 \mathrm{MHz}\right): \delta 1.19$ (d, $\left.J=6.2 \mathrm{~Hz}, 3 \mathrm{H}\right), 1.55(\mathrm{~m}, 2 \mathrm{H}), 3.37$ (dd, $J$ $=9.3$ and $7.0 \mathrm{~Hz}, 1 \mathrm{H}), 3.45(\mathrm{dd}, J=9.3$ and $3.8 \mathrm{~Hz}, 1 \mathrm{H}), 4.08(\mathrm{~m}, 2 \mathrm{H}), 4.55(\mathrm{~s}, 2 \mathrm{H}), 7.31(\mathrm{~m}$, $5 \mathrm{H}) ;{ }^{13} \mathrm{C} \mathrm{NMR}\left(\mathrm{CDCl}_{3}, 50 \mathrm{MHz}\right): \delta 23.8,40.9,68.1,71.3,73.4,74.4,127.7,127.8,128.5$, 137.8; MS (DCI / $\left.\mathrm{NH}_{3}\right): \mathrm{m} / \mathrm{z}=211[\mathrm{M}+\mathrm{H}]^{+}, 228\left[\mathrm{M}+\mathrm{NH}_{4}\right]^{+}$; IR (thin film): 3410, 3055, 3030, $2980,2930,2860,735,700 \mathrm{~cm}^{-1}$.

\section{Acknowledgements}

We thank Dr. R. Schmid (Hoffmann La Roche) for generous gift of $(R)$ - and $(S)$-MeO-BIPHEP: $(R)-(+)$ - and $(S)-(-)-6,6$ '-dimethoxy-2,2'-bis(diphenyl-phosphinoyl)-1,1'-biphenyl. O.L. is grateful to the Ministère de l'Education Nationale et de la Recherche for a grant (2001-2004). This work was partially supported by a grant from the CPER (2000-2006 action 10040 'Pôle Chimie du Vivant').

\section{References and Notes}

1. (a) Rychnovsky, S. D. Chem. Rev. 1995, 95, 2021. (b) Schneider, C. Angew. Chem. Int. Ed. 1998, 37, 1375.

2. For reviews on stereoselective synthesis of 1,3-diols, see (a) Bode, S. E.; Wolberg, M.; Müller, M. Synthesis 2006, 557. (b) Oishi, T.; Nakata, T. Synthesis 1990, 635.

3. (a) Ahmad, K.; Koul, S.; Taneja, S. C.; Singh, A. P.; Kapoor, M.; Riyaz-ul-Hassan; Verma, V.; Qazi, G. N. Tetrahedron: Asymmetry 2004, 15, 1685. (b) Ikeda, H.; Sato, E.; Sugai, T.; Ohta, H. Tetrahedron 1996, 52, 8113.

4. (a) Maier, G.; Roth, C.; Schmitt, R. K. Chem. Ber. 1985, 118, 704. (b) Maier, G.; Schmitt, R. K.; Seipp, U. Chem. Ber. 1985, 118, 722. (c) Bartoli, G.; Bosco, M.; Bellucci, M. C.; Dalpozzo, R.; Marcantoni, E.; Sambri, L. Org. Lett. 2000, 2, 45. (d) Ohtsuka, Y.; Kubota, T.; Ikeno, T.; Nagata, T.; Yamada, T. Synlett 2000, 535. 
5. Ru-catalysts: (a) Kitamura, M.; Ohkuma, T.; Inoue, S.; Sayo, N.; Kumobayashi, H.; Akutagawa, S.; Ohta, T.; Takaya, H.; Noyori, R. J. Am. Chem. Soc. 1988, 110, 629. (b) Kawano, H.; Ishii, Y.; Saburi, M.; Uchida, Y. J. Chem. Soc., Chem. Commun. 1988, 87. (c) Mezzetti, A.; Tschumper, A.; Consiglio, G. J. Chem. Soc. Dalton Trans. 1995, 49. (d) Brunner, H.; Terfort, A. Tetrahedron: Asymmetry 1995, 6, 919. (e) Blanc, D.; Ratovelomanana-Vidal, V.; Marinetti, A.; Genêt, J.-P. Synlett 1999, 480. (f) Marinetti, A.; Genêt, J.-P.; Jus, S.; Blanc, D.; Ratovelomanana-Vidal, V. Chem. Eur. J. 1999, 5, 1160. (g) Stritzke, K.; Schulz, S.; Nishida, R. Eur. J. Org. Chem. 2002, 3884. (h) Cossy, J.; Eustache, F.; Dalko, P. I. Tetrahedron Lett. 2001, 42, 5005. (i) Poss, C. S.; Rychnovsky, S. D.; Schreiber, S. L. J. Am. Chem. Soc. 1993, 115, 3360. (j) Mezzetti, A.; Consiglio, G. J. Chem. Soc., Chem. Commun. 1991, 1675. (k) Rychnovsky, S. D.; Griesgraber, G.; Zeller, S.; Skalitzky, D. J. J. Org. Chem. 1991, 56, 5161. (1) Pini, D.; Mandoli, A.; Iuliano, A.; Salvadori, P. Tetrahedron: Asymmetry 1995, 6, 1031; Ni-catalysts. (m) Ito, K.; Harada, T.; Tai, A.; Izumi, Y. Chem. Lett. 1979, 1049. (n) Ito, K.; Harada, T.; Tai, A.; Izumi, Y. Bull. Chem. Soc. Jpn. 1980, 53, 3367. (o) Sugimura, T.; Yoshikawa, M.; Yoneda, T.; Tai, A. Bull. Chem. Soc. Jpn. 1990, 63, 1080. (p) Tai, A.; Kikukawa, T.; Sugimura, T.; Inoue, Y.; Osawa, T.; Fujii, S. J. Chem. Soc., Chem. Commun. 1991, 795.

6. (a) Blandin, V.; Carpentier, J.-F.; Mortreux, A. Eur. J. Org. Chem. 1999, 1787. (b) Blandin, V.; Carpentier, J.-F.; Mortreux, A. Eur. J. Org. Chem. 1999, 3421. (c) Blandin, V.; Carpentier, J.-F.; Mortreux, A. New J. Chem. 2000, 24, 309. (d) Shao, L.; Seki, T.; Kawano, H.; Saburi, M. Tetrahedron Lett. 1991, 32, 7699. (e) Shao, L.; Kawano, H.; Saburi, M.; Uchida, Y. Tetrahedron 1993, 49, 1997.

7. Kumar, P.; Gupta, P.; Naidu, S. V. Chem. Eur. J. 2006, 12, 1397.

8. (a) Narasaka, K.; Pai, F.-C. Chem. Lett. 1980, 1415. (b) Narasaka, K.; Pai, F.-C. Tetrahedron 1984, 40, 2233.

9. (a) Chen, K.-M.; Gunderson, K. G.; Hardtmann, G. E.; Prasad, K.; Repic, O.; Shapiro, M. J. Chem. Lett. 1987, 1923. (b) Chen, K.-M.; Hardtmann, G. E.; Prasad, K.; Repic, O.; Shapiro, M. J. Tetrahedron. Lett. 1987, 28, 155. (c) Yamashita, H.; Narasaka, K. Chem. Lett. 1996, 539.

10. (a) Evans, D. A.; Chapman, K. T. Tetrahedron Lett. 1986, 27, 5939. (b) Evans, D. A.; Chapman, K. T.; Carreira, E. M. J. Am. Chem. Soc. 1988, 110, 3560.

11. Recent references (a) Leroux, R.; Desroy, N.; Phansavath, P.; Genêt, J.-P. Synlett 2005, 429. (b) Labeeuw, O.; Phansavath, P.; Genêt, J.-P. Tetrahedron: Asymmetry 2004, 15, 1899. (c) Mordant, C.; Reymond, S.; Ratovelomanana-Vidal, V.; Genêt, J.-P. Tetrahedron 2004, 60, 9715. (d) Mordant, C.; Cano de Andrade, C.; Touati, R.; Ratovelomanana-Vidal, V.; Ben Hassine B.; Genêt, J.-P. Synthesis 2003, 2405. (e) Ratovelomanana-Vidal, V.; Girard, C.; Touati, R.; Tranchier, J.-P.; Ben Hassine B.; Genêt, J.-P. Adv. Synth. Catal. 2003, 345, 261.

12. For reviews on ruthenium-mediated hydrogenation reactions, see (a) Noyori, R.; Ohkuma, T. Angew. Chem. Int. Ed. 2001, 40, 40. (b) Ohkuma, T.; Kitamura, M.; Noyori, R. Catalytic 
Asymmetric Synthesis; Ojima, I., Ed.; Wiley: VCH, 2000, 1. (c) Genêt, J.-P. Acc. Chem. Res. 2003, 36, 908.

13. Madec, J.; Pfister, X.; Phansavath, P.; Ratovelomanana-Vidal, V.; Genêt, J.-P. Tetrahedron 2001, 57, 2563.

14. Genêt, J.-P.; Pinel, C.; Ratovelomanana-Vidal, V.; Mallart, S.; Caño de Andrade, M. C.; Laffitte, J. A. Tetrahedron: Asymmetry 1994, 5, 665.

15. Rodriguez, S.; Kayser, M. M.; Stewart, J. D. J. Am. Chem. Soc. 2001, 123, 1547.

16. Kiyooka, S.; Shahid, K. A. Bull. Chem. Soc. Jpn. 2001, 74, 1485.

17. Kamal, A.; Ramesh Khanna, G. B.; Ramu, R. Tetrahedron: Asymmetry 2002, 13, 2039.

18. Evans, D. A.; Murry, J. A.; Kozlowski, M. C. J. Am. Chem. Soc. 1996, 118, 24.

19. (a) Nahm, S.; Weinreb, S. M. Tetrahedron Lett. 1981, 22, 3815. (b) Iseki, K.; Asada, D.; Kuroki, Y. J. Fluorine Chem. 1999, 97, 85.

20. Schmid, R.; Foricher, J.; Cereghetti, M.; Schönholzer, P. Helv. Chim. Acta 1991, 74, 370.

21. (a) Duprat de Paule, S.; Jeulin, S.; Ratovelomanana-Vidal, V.; Genêt, J.-P.; Champion, N.; Dellis, P. Tetrahedron Lett. 2003, 44, 823. (b) Duprat de Paule, S.; Jeulin, S.; Ratovelomanana-Vidal, V.; Genêt, J.-P.; Champion, N.; Dellis, P. Eur. J. Org. Chem. 2003, 1931. (c) Duprat de Paule, S.; Jeulin, S.; Ratovelomanana-Vidal, V.; Genêt, J.-P.; Champion, N.; Deschaux, G.; Dellis, P. Org. Process Res. Dev. 2003, 7, 399.

22. Labeeuw, O.; Blanc, D.; Ratovelomanana-Vidal, V.; Phansavath, P.; Genêt, J.-P. Eur. J. Org. Chem. 2004, 2352. 